\title{
Can diffusion tensor imaging predict cerebral palsy in term neonates with hypoxic ischemic encephalopathy?
}

\author{
Ahmed A. ElBeheiry ${ }^{1 *}$ D, Mohamed A. Elgamal', Ashraf N. Ettaby ${ }^{1}$, Tarek E. Omar ${ }^{2}$ and Adham O. Badeib²
}

\begin{abstract}
Background: Hypoxic ischemic encephalopathy (HIE) is a principal cause of pediatric mortality and morbidity, with possible neurologic sequel, such as cerebral palsy. Thus, it is of outmost importance to assess, shortly after birth, the prognosis of neonates with HIE. The purpose of this study was to assess the role of diffusion tensor imaging (DTI) in early prediction of cerebral palsy in term neonates with HIE. The study was carried out initially on 35 full-term neonates admitted to the neonatal intensive care unit (NICU) with manifestations of HIE. Imaging was done at the age of $10.32 \pm 1.78$ days including conventional MRI and DTI (for detection of white matter injury). Thirty-three infants out of the initially examined 35 were finally evaluated at the age of 1 year with the Bayley Scales of Infant and Toddler Development 3rd edition for development of cerebral palsy. Another MRI brain study was done at 1 year of age to evaluate the final brain imaging features and assess white matter integrity.

Results: Seventeen infants (17/33) were clinically normal while $16(16 / 33)$ acquired cerebral palsy (CP) by the end of the first year. Initial conventional MRI showed false-negative results in 7 patients. Diffusion tensor imaging showed significantly different reduction in fractional anisotropy within the examined brain regions of interest (ROls) between CP and normal outcome infants mostly at the posterior limb of the internal capsule, centrum semiovale, and corpus callosum with significant cutoff values of $\leq 0.435, \leq 0.235$, and $\leq 0.45$, respectively.
\end{abstract}

Conclusions: Diffusion tensor imaging is a valuable tool for early prediction of CP in HIE neonates.

Keywords: Hypoxic ischemic encephalopathy, Cerebral palsy, Diffusion tensor imaging

\section{Background}

Hypoxic ischemic encephalopathy (HIE) is a major cause of neurologic disabilities in term neonates. The incidence of HIE ranges from 1 to 8 per 1000 live births in developed countries and is as high as 26 per 1000 live births in underdeveloped countries [1].

Hypoxic ischemic encephalopathy (HIE) is considered one of the main causes of morbidity and mortality among the neonatal age group, carrying the potential of causing long-lasting neurological damage such as cerebral palsy (CP), convulsions, and mental retardation [2].

Brain cooling and therapeutic hypothermia is considered the treatment of choice in these hypoxic neonates

\footnotetext{
* Correspondence: elbeheiryahmed@gmail.com

'Department of Radiodiagnosis, Faculty of Medicine, University of Alexandria, Alexandria, Egypt

Full list of author information is available at the end of the article
}

due to its neuroprotective effect especially in cases of moderate and severe HIE [3].

After applying neuroprotective measures, evaluation of the degree of brain injury is of extreme importance in guiding further neurological care. These data are used to plan adjuvant therapy and rehabilitative procedures and to supply information regarding the future prognostic outcomes [4].

Both objective and accurate markers are needed to assess the prognosis of HIE in full-term neonates. Although ultrasonography (US), computed tomography (CT), and magnetic resonance imaging (MRI) can be used to evaluate brain injury, still they are unable to provide a quantitative data regarding the degree of affection of the brain mainly white matter which is very important to reach an accurate prognosis $[5,6]$.

Conventional MRI usually reveals two main types of brain injury in neonates with HIE; these are abnormalities 
detected at the thalami and basal ganglia (central pattern) as well as damage at the watershed zones of the brain (peripheral pattern). These findings express obvious correlation with late cognitive and motor abnormalities [7]. However, conventional MRI done in first days of life may miss significant brain injuries (especially after brain cooling) with highest diagnostic sensitivity at about $1-2$ weeks postnatal [8].

Due to this limitation, conventional MRI should be interpreted with other advanced techniques including diffusion tensor imaging (DTI) and magnetic resonance spectroscopy (MRS) [9].

The DTI is a modern advanced technique derived from modification of diffusion weighted imaging (DWI). DTI has proved outstanding performance in evaluating the direction and integrity of white matter tracts of the brain [10].

Estimation of values of fractional anisotropy (FA) by DTI within multiple areas in the brain can provide both quantitative and qualitative data concerning the white matter tracts, can assess white matter abnormalities, and can also provide useful data about white matter repair. Currently, it is believed that DTI can be a valuable tool in the assessment of HIE-related white matter injury providing accurate information concerning the severity and prognosis of the condition [11].

The aim of this study was to assess the additive role of DTI over conventional MRI in early prediction of cerebral palsy in term neonates with hypoxic ischemic encephalopathy.

\section{Methods}

Approval for this cross-sectional prospective study was obtained from the local human research ethics committee. All study procedures were carried out in accordance with the Declaration of Helsinki regarding research involving human subjects [12]. Written informed consent was obtained from the parents of each child.

\section{Patients}

From September 2015 to March 2016, full-term neonates admitted to the neonatal intensive care unit (NICU) with HIE ranging in severity from moderate to profound according to Sarnat's scoring [13] were referred to the Radiodiagnosis Department for brain imaging. The HIE score based on Sarnat's scoring system is a clinical tool comprising of a set of clinical signs associated with CNS dysfunction that is used to assess the status of a child following birth asphyxia. Depending on the neurobehavioral signs, neonates are divided into stages I, II, and III (mild, moderate, and severe, respectively) [13].

Inclusion criteria included (a) full-term neonates (37-42 weeks gestational age) (b) exposed to perinatal hypoxic conditions based on history of difficult or prolonged labor, placenta previa, cord prolapse, and/ or severe hemorrhage; the perinatal period commences from the 28th week of gestation to the 1st 7 days following delivery. Pre-term neonates (less than 37 weeks gestational age) and neonates with suspected neuro-metabolic diseases, neonatal sepsis, traumatic birth injuries, kernicterus, and congenital brain malformations were excluded. Cases with no available follow-up imaging after 1 year were also excluded.

The study initially included 35 neonates: 19 males and 16 females with mean gestational age of $39.0 \pm 2.0$ weeks and mean birth weight of $3470.3 \pm 500.8 \mathrm{~g}$. Apgar score done at $5 \mathrm{~min}$ ranged from 2 to 7 . The patients' conditions tended to be unstable in the first week, so initial MRI was conducted at the age of 10-14 days with mean age of $10.32 \pm 1.78$ days. All neonates included in the study underwent initial imaging protocol including conventional MRI and diffusion tensor imaging (DTI). All neonates received brain cooling in the first 3 days of their lives (which is considered a routine protocol done in the NICU of our tertiary care referring hospital) prior to doing the initial MRI examinations.

Owing to loss of contact with their parents shortly after the first examination, two patients were discarded from our study, so 33 patients out of the initially examined 35 neonates were evaluated clinically and by imaging after 1 year from the first exam (from September 2016 to March 2017) for development of cerebral palsy.

\section{MRI acquisition protocol}

Examination was done on $1.5 \mathrm{~T}$ GE Signa machine (General Electric Healthcare, Piscataway, NJ). MR images were acquired with the following protocols:

(a) Conventional MRI protocol included 3D T1weighted sequences (repetition time (TR) 6.6, echo time (TE) 2.6, inversion time (TI) 600, number of excitations (NEX) 1, flip angle 25, matrix $192 \times 192,1.2 \mathrm{~mm}$ slice thickness, with 0 $\mathrm{mm}$ gap) acquired in an axial plane and reconstructed in coronal and sagittal planes, fast spin echo (FSE) T2-weighted axial sequences (TR 4400, TE 120, NEX 2, matrix $384 \times 320,3 \mathrm{~mm}$ slice thickness, with $1.0 \mathrm{~mm}$ gap), and diffusionweighted imaging (DWI) with single shot, $b$ value $=1000 \mathrm{~mm}^{2} / \mathrm{s}$ (TR 8000, TE 78.8, NEX 1, field of view (FOV) $220 \times 220$, matrix $140 \times 140$, slice thickness $2.5 \mathrm{~mm}$ with $0.6 \mathrm{~mm}$ gap).

(b) DTI acquired with pulsed field gradients in 21 directions with single-shot echo-planar imaging (TR, $7800 \mathrm{~ms}$; TE, $102 \mathrm{~ms} ; 40$ sections; $b$ value, 0 / $1000 \mathrm{~s} / \mathrm{mm}^{2}$; 3-mm sections, no gap; FOV, $18 \times 36$ $\mathrm{cm}$; and $128 \times 256$ acquisition matrix). 


\section{MRI data analysis and imaging evaluation}

MRI data were reviewed for each neonate blinded to the clinical data apart from suspicion for HIE. The following steps of imaging evaluation were followed:

a. MRI assessment for signs of HIE which included the following:

1. Signs of acute profound HIE (severe hypotension)-loss of T1 hyperintensity of the posterior limb of the internal capsule (PLIC) with restricted diffusion within the metabolically active areas of the brain including the ventrolateral thalami, posterior putamina, perirolandic regions, and corticospinal tracts (CST) along the anterior aspect of the brain stem as well as the hippocampi [14].

2. Signs of partial prolonged HIE (mild to moderate hypotension)-loss of gray-white matter differentiation with restricted diffusion of the cortex and subcortical white matter along the intervascular watershed areas between the anterior and middle as well as middle and posterior cerebral arterial territories [14].

b. DTI assessment (steps were done on GE workstation "Advantage 4.6") for signs of white matter injury:

(a) Generation of fractional anisotropy maps by registration of DTI images to the non-diffusion volume in order to reduce distortion sequel to eddy currents followed by removal of any extracerebral tissues by brain extraction process.

(b) Analysis of the FA maps using ROI approach where multiple (around $3 \mathrm{~mm}$ ) regions of interest (ROIs) were placed on the PLIC, the supraganglionic white matter mainly within the centrum semiovale, optic radiations, and occipital white matter (OWM) bilaterally.

\section{Outcome assessment \\ Imaging assessment}

Follow-up imaging after 1 year from the first examination included the following:

1. Conventional MRI including 3D T1, axial T2, and axial FLAIR sequences.

2. Diffusion tensor imaging (DTI) with tractography to identify the integrity of white matter mainly the corticospinal tracts (along their course from centrum semiovale through PLIC and down to the brain stem) which are the main tracts affected in cases of cerebral palsy.

\section{Clinical assessment}

Sensori-neural and psychomotor integrity was assessed clinically by using the Bayley Scales of Infant and Toddler Development, 3rd edition (Bayley III scale) [15], performed by an expert pediatric neurologist. The Bayley scale is the most widely used tool which assesses developmental functioning in infants and young children between 1 month and 42 months. The Bayley III scale is based upon assessment of three major areas of development including cognitive scale, communication or language scale, and motor scale. The outcome of HIE is thus graded, according to the cognitive, language, and motor composites, into normal (v. good or good outcome $=$ Bayley III score $\geq 85$ in all 3 domains) and adverse outcome (fair or poor $=$ Bayley III score $<85$ in any domain). The primary purposes of Bayley III are to identify children with developmental delay and to provide information for intervention planning [15].

\section{Statistical methodology}

Data were collected and entered to the computer using SPSS (Statistical Package for Social Science) program for statistical analysis (ver 21). Data were entered as numerical or categorical, as appropriate. The KolmogorovSmirnov test of normality revealed significance in the distribution of some variables, so the non-parametric statistics was adopted. Data were described using minimum, maximum, mean, standard deviation, and 95\% confidence interval $(\mathrm{CI})$ of the mean for the normally distributed data. Data were described using minimum, maximum, median, and interquartile range for notnormally distributed data. Comparisons were carried out between two studied independent not-normally distributed subgroups using the Mann-Whitney $U$ test. Comparisons were carried out between more than two studied independent not-normally distributed subgroups using the Kruskal-Wallis test. Chi-square test was used to test the association between qualitative variables. Area under the ROC (AUC) curve was carried using MedCalc Software version 14. Comparison of area under the ROC (AUC) curves was done using Delong's method. Percentage of agreement was assessed by kappa coefficient. An alpha level was set to $5 \%$ with a significance level of $95 \%$, and a beta error accepted up to $20 \%$ with a power of study of $80 \%$.

\section{Results}

\section{Demographic data}

Seventeen neonates $(51.5 \%)$ were males while the remaining 16 (48.4\%) were females. Among the studied 
neonates, $18(54.54 \%)$ were born by cesarean section (CS) while the other 15 (45.45\%) were born through normal vaginal delivery (NVD). According to severity, 21 cases $(63.6 \%)$ were Sarnat stage II or moderate degree of HIE, while the remaining 12 cases (36.3\%) were Sarnat stage III or severe degree of HIE (Table 1).

\section{Final outcome (clinical and radiological outcome) at the} age of 1 year (the golden reference)

Seventeen infants were clinically normal at the end of the first year, all of whom showed normal follow-up MRI examinations while the other 16 infants acquired cerebral palsy: 6 of whom showed MRI central pattern of affection with involvement of the thalami, putamina, and posterior limbs of the internal capsules (having clinically dystonic $\mathrm{CP}$ ) and the other 10 showed peripheral pattern of injury with periventricular leukomalacia (having clinically spastic $\mathrm{CP}$ ).

\section{Initial conventional MRI and DWI findings as compared to} the final outcome

Initial conventional MRI was normal in 24 neonates, 17 of whom proved on follow-up after 1 year to have normal outcome both clinically and radiologically, while the other 7 showed false-negative results as they developed spastic CP by 1 year with their follow-up MRIs showing periventricular leukomalacia.

Conventional MRI was initially abnormal in the other 9 neonates, 6 showed central pattern of affection (T1 hyperintensity of the putamina and thalami with loss of $\mathrm{T} 1$ hyperintensity of the posterior limbs of the internal capsules), proved on follow-up to have dystonic $\mathrm{CP}$, while the other 3 neonates showed watershed pattern with smudging of the gray-white matter interface at the watershed regions bilaterally and proved on follow-up to have PVL and spastic CP. The DWI was normal for all neonates at the time of examination.

Table 1 Distribution of the studied neonates according to demographic data, mode of delivery, and HIE Sarnat staging $(n=33)$

\begin{tabular}{lll}
\hline & Number & Percentage \\
\hline Sex & 17 & 51.5 \\
Male & 16 & 48.4 \\
Female & & \\
Delivery & 18 & 54.54 \\
CS & 15 & 45.45 \\
NVD & & \\
HIE Sarnat staging & 21 & 63.6 \\
Stage II & 12 & 36.3 \\
Stage III &
\end{tabular}

CS cesarean section, NVD normal vaginal delivery
Thus, the initial conventional MRI showed sensitivity of $56.25 \%$, specificity of $100 \%$, positive predictive value (PPV) of $100 \%$, negative predictive value (NPV) of $70.83 \%$, accuracy of $78.79 \%$, and overall moderate agreement with follow-up reaching $57 \%$ (Table 2).

\section{Initial DTI results as compared to the final outcome}

FA values were lower in patients with $\mathrm{CP}$ outcome compared to those with normal outcome in nearly most ROIs as shown in Table 3. The FA values were significantly lower within the PLIC in patients with dystonic $\mathrm{CP}$ outome. Spastic CP patients also showed significantly lower FA within the centrum semiovale. Corpus callosum genu and splenium FA values also showed significant lower FA values in CP outcome patients as compared to normal outcome infants. No signifcant difference was noted as regards FA values within the optic radiations as well as the occipital white matter (OWM).

ROC curve analysis was performed to study the significant cutoff values of FA discriminating between CP and normal outcome showing centrum semiovale cutoff value $\leq 0.235$, and genu and splenium of the corpus callosum cutoff value $\leq 0.45$ with best significance being PLIC cutoff value $\leq 0.435$ (Table 4 and Fig. 1).

All seven neonates with false-negative initial conventional MRI results had significant reduction of FA values (below the aforementioned cutoff values) within the measured white matter regions.

Example for the difference in DTI/FA between those with normal and CP outomes is shown in Fig. 2, 3, and 4.

\section{Discussion}

Perinatal hypoxia in full-term neonates is a vital cause of long-term neurologic complications ranging from mild behavioral deficits to intractable seizures, mental retardation, and cerebral palsy. With improvements in care of at-risk neonates, more and more children survive. Thus, it is increasingly important to evaluate, soon after birth, the prognosis of neonates with perinatal hypoxic ischemic encephalopathy [16].

In order to answer the question of the title of the study and understand the role of advanced MRI techniques mainly DTI in the prognosis of perinatal HIE, the current study tried to find an answer for the following questions: Are conventional MRI sequences and DWI sufficient for early identification of perinatal HIE and prediction of cerebral palsy? Does DTI have a role in diagnosis and prognosis of perinatal HIE? And lastly, what is the recommended imaging roadmap in cases of perinatal HIE?

Taking into consideration the first question (Are conventional MRI sequences and DWI sufficient for early identification of perinatal HIE and prediction of cerebral palsy?), the current study showed that although MRI is 
Table 2 Agreement of the initial routine MRI with final outcome

\begin{tabular}{|c|c|c|c|c|c|c|c|c|c|}
\hline & & \multicolumn{2}{|c|}{ Outcome at 1 year } & \multirow[t]{2}{*}{ Sensitivity } & \multirow[t]{2}{*}{ Specificity } & \multirow[t]{2}{*}{ PPV } & \multirow[t]{2}{*}{ NPV } & \multirow[t]{2}{*}{ Accuracy } & \multirow[t]{2}{*}{ Agreement } \\
\hline & & Normal & Abnormal $^{@}$ & & & & & & \\
\hline \multirow[t]{2}{*}{ Initial routine MRI } & Normal & 17 & 7 & 56.25 & 100 & 100 & 70.83 & 78.79 & 57\% (kappa 0.57) \\
\hline & Abnormal & 0 & 9 & & & & & & \\
\hline$x^{2}(p)$ & & \multicolumn{2}{|c|}{$13.148^{*}\left(<0.001^{*}\right)$} & & & & & & \\
\hline
\end{tabular}

Kappa coefficient was used for percentage of agreement

$X^{2}(p) X^{2}$ and $p$ values for chi square test for comparing between the two categories, NPV negative predictive value, $P P V$ positive predictive value

*Statistically significant at $p<0.05$

${ }^{\circledR}$ Abnormal outcome was assessed both clinically as cerebral palsy and by MRI as different patterns of brain injury

still the study of choice in cases of perinatal HIE, yet its main role is evident in cases of acute profound insult with deep gray matter affection pattern. This was clearly evident in the current study as all cases with such pattern (six cases) were accurately detected within the neonatal period and they turned out to have $\mathrm{CP}$ by the end of their first year.

On the other hand, conventional MRI as well as DWI showed poor results with high false-negative cases in neonates with mild to moderate hypoperfusion, as seven out of the ten cases $(70 \%)$ who turned to have periventricular leukomalacia at the end of the first year showed initial normal MRI with even no evidence of diffusion restriction.

Explanation for such false-negative results of conventional MRI and DWI was found in previous studies of Agut et al. [17] and Hayakawa et al. [18], who studied early MRI features of 40 and 17 full-term neonates, respectively, and concluded that despite the higher sensitivity of DWI compared to conventional MRI in the characterization of brain injury in the acute phase of HIE, yet DWI shows restricted diffusion with decrease of ADC values only in the first hours after the anoxic-ischemic event. Low ADC values tend to progressively increase in the first week after the event, resulting in a pseudonormalization of values between days 4 and 6 . Being strictly dependent on the timing of the scan, both studies $[17,18]$ have demonstrated that the diagnostic and prognostic values of $\mathrm{ADC}$ in the subacute stage of HIE are significantly decreased.

This was clearly found in the current study as all neonates were imaged around the tenth day of life, the time of which pseudonormalization of ADC was expected. Such fact resulted in normal appearance of DWI in all cases and lead to reduction in sensitivity, negative predictive value, and overall accuracy of routine MRI (including conventional MRI and DWI) to $56.25 \%, 70.83 \%$, and $78.79 \%$, respectively. In other words, conventional MRI sequences and DWI are likely not sufficient alone in early diagnosis of all cases of perinatal HIE especially in cases with moderate hypoperfusion or partial prolonged insult.

"Does DTI have role in diagnosis and prognosis of perinatal HIE?" was the second question for which we tried to find an answer.

DTI carries extra information about directional diffusivity (both longitudinal and radial) and fractional anisotropy (FA) which can improve the detection of disruption of white matter tracks. It is thought that reduced FA values are linked to cell death and loss of structural components of white matter fibers. Rutherford et al. [19] reported that apoptosis was more noticeable than necrosis in children in the subacute stage of HIE, which could explain why FA values decreased, but ADC values were normal.

Our results verified that FA values had high diagnostic and prognostic values. In particular, FA values were

Table 3 Fractional anisotropy (FA) values in newborns compared with final outcome after 1 year

\begin{tabular}{|c|c|c|c|c|c|c|c|c|}
\hline \multirow{3}{*}{$\begin{array}{l}\text { Outcome at } 1 \\
\text { year }\end{array}$} & \multicolumn{8}{|c|}{ FA values of the newborns at first examination } \\
\hline & \multicolumn{2}{|c|}{ Centrum semiovale } & \multicolumn{2}{|l|}{ PLIC } & \multirow[t]{2}{*}{ Genu of CC } & \multirow{2}{*}{$\begin{array}{l}\text { Splenium of } \\
\text { CC }\end{array}$} & \multirow[t]{2}{*}{ OWM } & \multirow[t]{2}{*}{ Optic radiation } \\
\hline & Right & Left & Right & Left & & & & \\
\hline $\begin{array}{l}\text { Normal } \\
\text { outcome (17) }\end{array}$ & $0.27 \pm 0.02$ & $0.28 \pm 0.03$ & $0.45 \pm 0.02$ & $0.46 \pm .02$ & $0.54 \pm 0.02$ & $0.56 \pm 0.02$ & $0.23 \pm 0.01$ & $0.30 \pm 0.01$ \\
\hline $\begin{array}{l}\text { Cerebral palsy } \\
\text { (16) }\end{array}$ & $0.18 \pm 0.04$ & $0.20 \pm 0.04$ & $0.35-0.03$ & $0.36 \pm 0.04$ & $0.45 \pm 0.08$ & $0.46 \pm 0.09$ & $0.22 \pm 0.04$ & $0.29 \pm 0.04$ \\
\hline $\begin{array}{l}\text { Test of } \\
\text { significance, } p \\
\text { value }\end{array}$ & $\begin{array}{l}Z_{(\mathrm{MW})}=4.304 \\
p=0.000^{*}\end{array}$ & $\begin{array}{l}Z_{(\mathrm{MW})}=4.022 \\
p=0.000^{*}\end{array}$ & $\begin{array}{l}Z_{(\mathrm{MM})}=4.862 \\
p=0.000^{*}\end{array}$ & $\begin{array}{l}Z_{(\mathrm{MM})}=4.378 \\
p=0.000^{*}\end{array}$ & $\begin{array}{l}Z_{(\mathrm{MW})}=2.937 \\
p=0.003^{*}\end{array}$ & $\begin{array}{l}Z_{(\mathrm{MM})}=3.112 \\
p=0.002^{*}\end{array}$ & $\begin{array}{l}Z_{(\mathrm{MW})}=0.605, p \\
=0.576 \mathrm{NS}\end{array}$ & $\begin{array}{l}Z_{(\mathrm{MW})}=0.805, p \\
=0.455 \mathrm{NS}\end{array}$ \\
\hline
\end{tabular}

Data are expressed as mean (SD), Mann-Whitney $U$ test

CC corpus callosum, FA fractional anisotropy, OWM occipital white matter, PLIC posterior limb of internal capsule, NS statistically not significant ( $p>0.05$ )

*Statistically significant $(p<0.05)$ 
Table 4 ROC curve analysis of DTI/FA values for discrimination of occurrence of cerebral palsy

\begin{tabular}{|c|c|c|c|c|c|c|c|}
\hline Index & $\mathrm{AUC}(95 \% \mathrm{Cl})$ & $Z$ ( $p$ value $)$ & Cutoff value $^{(Y l)}$ & Sensitivity $(95 \% \mathrm{Cl})$ & Specificity $(95 \%$ Cl) & PPV (\%) & NPV (\%) \\
\hline Centrum semiovale & $0.949(0.811-0.995)$ & $9.275\left(<0.0001^{*}\right)$ & $\leq 0.235$ & 93.75 (69.8-99.8) & $94.12(71.3-99.9)$ & 93.7 & 94.1 \\
\hline PLIC & $1.000(0.894-1.000)$ & $0\left(<0.0001^{*}\right)$ & $\leq 0.435$ & $100.00(79.4-100.0)$ & $100.00(80.5-100.0)$ & 100.0 & 100.0 \\
\hline OWM & $0.581(0.397-0.750)$ & 0.789 (0.4303 NS) & & & & & \\
\hline OR & $0.511(0.332-0.688)$ & 0.103 (0.9182 NS) & & & & & \\
\hline Genu of CC & $0.811(0.637-0.925)$ & $4.107\left(<0.0001^{*}\right)$ & $\leq 0.45$ & 56.25 (29.9-80.2) & $100.00(80.5-100.0)$ & 100.00 & 70.8 \\
\hline Splenium of CC & $0.814(0.637-0.929)$ & $3.911\left(<0.0001^{*}\right)$ & $\leq 0.45$ & $60.00(32.3-83.7)$ & $100.00(80.5-100.0)$ & 100.0 & 73.9 \\
\hline
\end{tabular}

AUC area under the curve, $C C$ corpus callosum, $C$ confidence interval, NPV negative predictive value, OR optic radiation, OWM occipital white matter, $P L I C$ posterior limb of internal capsule, PPV positive predictive value, NS statistically not significant $(p>0.05)$

*Statistically significant $(p<0.05)$

remarkably decreased in most of the dense white matter tracts, mainly the corpus callosum and posterior limbs of the internal capsules (PLIC) as well as the centrum semiovale (CSO). Significant differences in FA values were detected between newborns with $\mathrm{CP}$ outcome and those with normal outcome with detectable cutoff values of FA within the corpus callosum, PLIC, and CSO averaging $\leq 0.45, \leq 0.435$, and $\leq$ 0.235 , respectively. Significant predictive power of FA within the PLIC had the highest value of accuracy (AUC 1.0) showing 100\% sensitivity, specificity, and negative and positive predictive values. Again, all neonates with normal appearing MRI who developed periventricular leukomalacia later on (false-negative

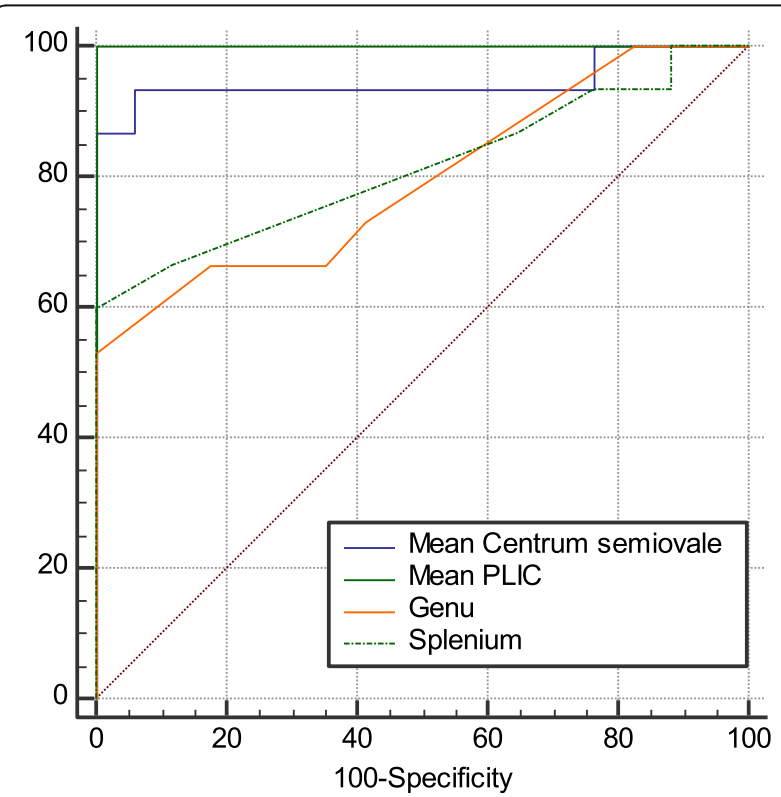

Fig. 1 ROC curve analysis for FA of PLIC (continuous green line), CSO (blue line), and genu and splenium of the corpus callosum (red and dotted green lines, respectively) where FA of PLIC $\leq 0.435$ was shown to be the most statistically significant discriminator of occurrence of cerebral palsy with area under the ROC curve (AUC) $=$ 1.000 with ${ }^{*} p<0.0001$ cases) showed initially abnormally reduced FA values within the examined brain regions.

Diagnostic accuracy of DTI in neonatal HIE was previously documented by Barkovich et al. [20], who studied sequentially ten neonates with encephalopathy within the first 2 weeks of life and found that DTI-derived parameters were consistently abnormal even at times where MRI were completely unremarkable.

Similar results were also obtained by Ward et al. [21], in 20 neonates with HIE as they observed that FA stayed reduced, while ADC pseudonormalized with time. At the same time, the authors stated that FA decreased not only in severe basal ganglia and white matter injury but reduced in all moderate basal ganglia and white matter injuries. The authors' assumption was that FA represents an important marker in neonates, mainly in moderate HIE predominantly when results of ADC values are normal.

As regards the prognostic role of DTI, the current results were also matching with the previous study of Brissaud et al. [22], who studied 22 full-term neonates with HIE and found that FA values in PLIC areas showed best correlation with outcome, where the lower the FA values in the PLIC, the worse the infants' neurologic outcome. Cutoff value for FA within the PLIC in their study was 0.38 (lower than the cutoff in the current study).

The only explanation which we found for such difference in FA values in the PLIC is brain cooling application which was adopted in all of our examined neonates, as a routine protocol done in the NICU for neonates with moderate or severe perinatal HIE, while not routinely mentioned in the other studies. Brain cooling is a neuroprotective therapy that reduces the extent of brain injury via at least the following three mechanisms. Firstly, hypothermia results in a graded reduction in cerebral metabolism that reduces accumulation of excitotoxic neurotransmitters, and suppresses oxygen free radical release. Secondly, it has a particular role in suppressing apoptotic processes in the developing brain (i.e., programmed cell death). Thirdly, there is good evidence that cooling can suppress the release of pro- 


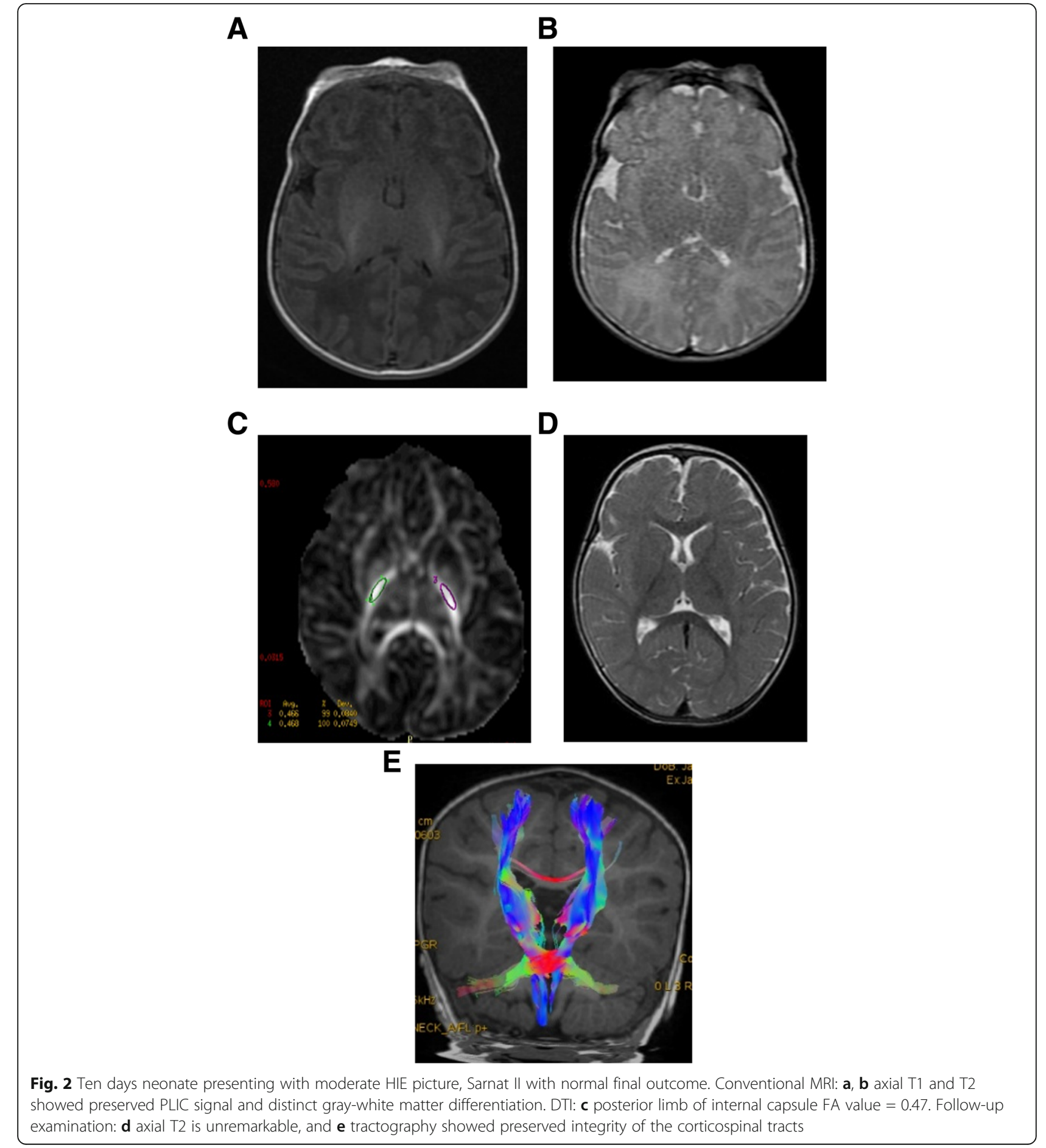

B

inflammatory cytokines and interleukins, reducing direct neurotoxicity, such via suppression of microglial activation [23].

We believe that brain cooling has protective effect on FA of white matter, limiting its damage, as mentioned before in the study of Chan et al. [24], regarding effect of brain cooling as a neuroprotective method on alteration of brain metabolites on MR spectroscopy.
"What is the recommended imaging roadmap in cases of perinatal HIE?" was our last question. From the aforementioned results, we suggest that conventional MRI with DWI is sufficient for early identification as well as prediction of development of CP in cases of acute profound hypoxia with central pattern of affection. In cases with normal appearing MRI and suspected mild to moderate HIE, doing DTI with emphasis of accurate 

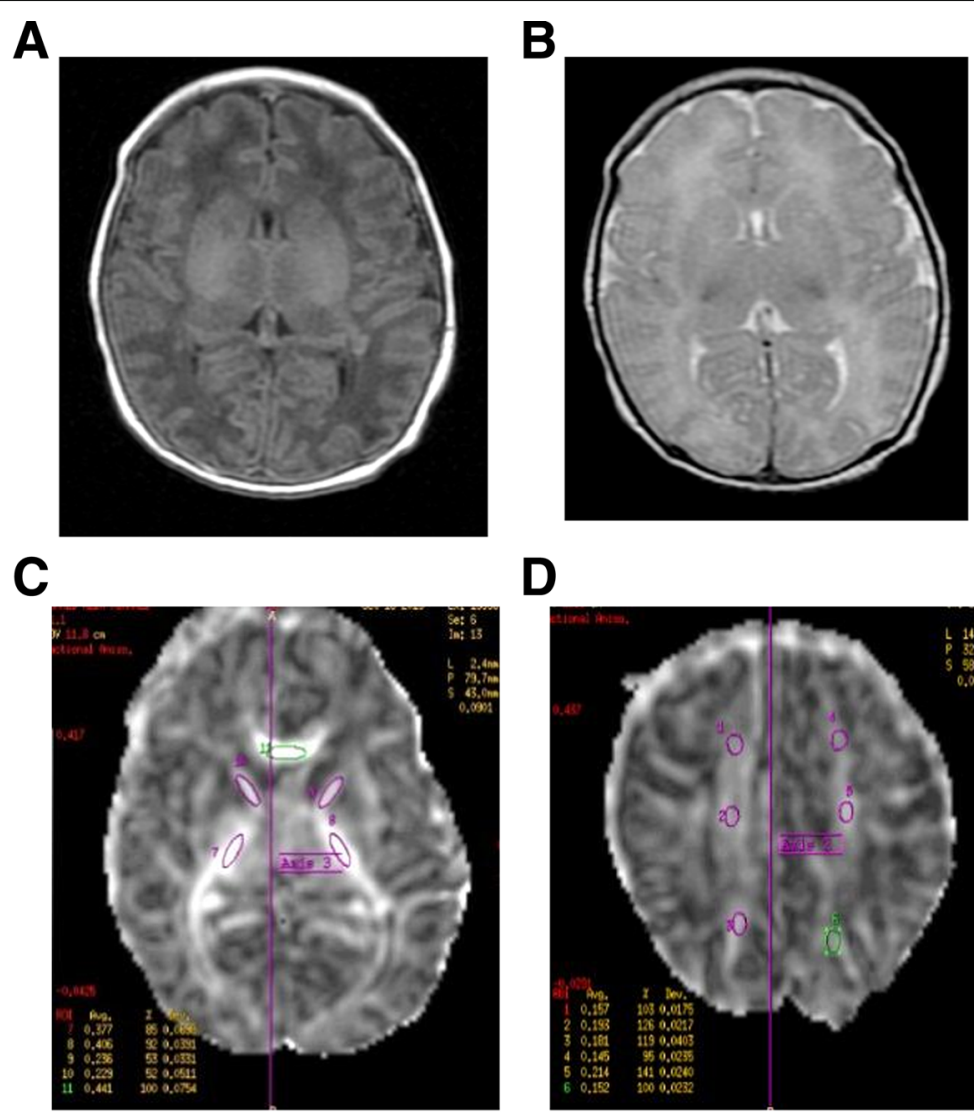

D
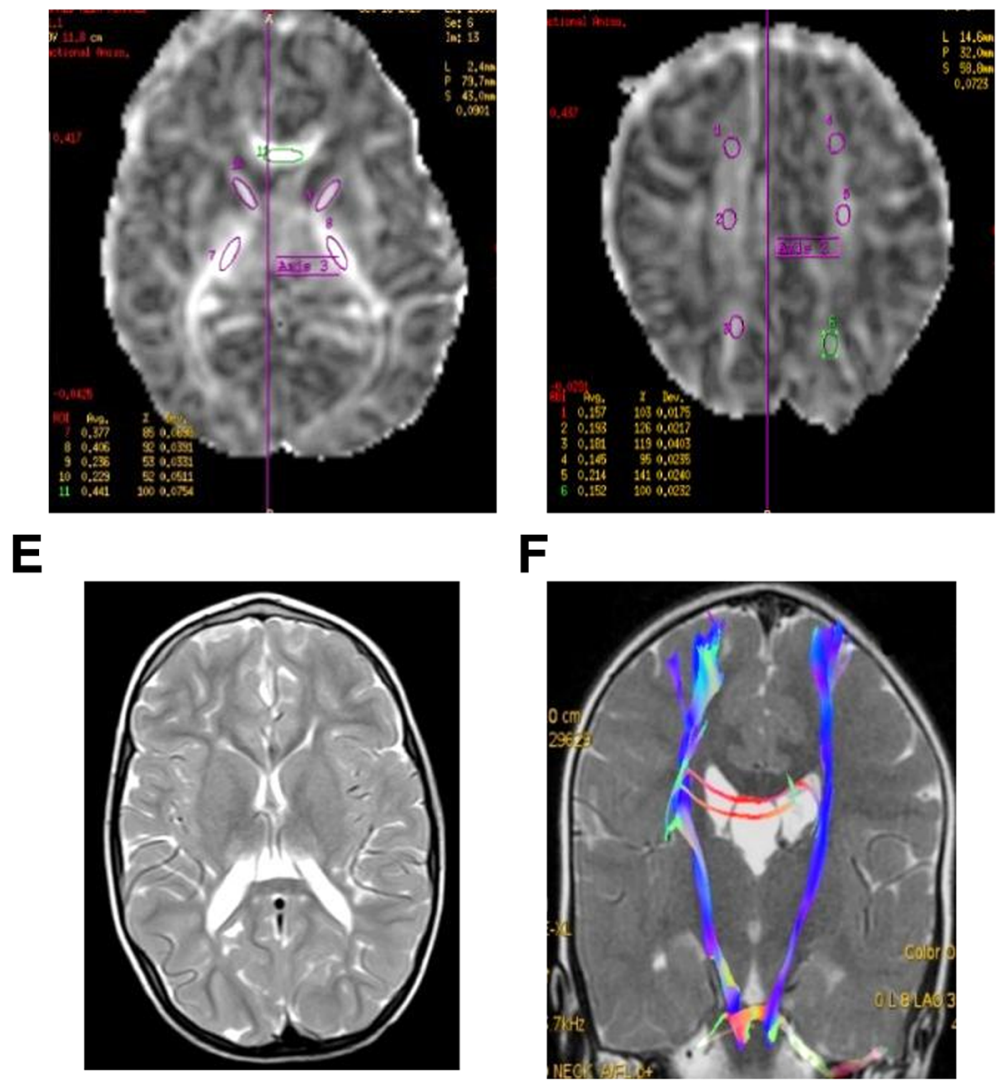

F

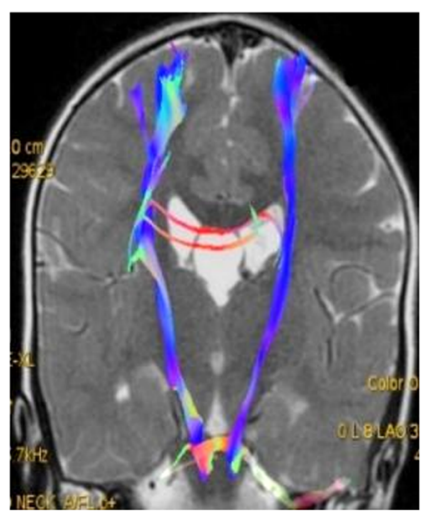

Fig. 3 Nine days neonate presenting with severe HIE picture, Sarnat III with CP outcome. Conventional MRI: a, b axial T1 and T2 showed preserved PLIC signal and distinct gray-white matter differentiation. DTI: $\mathbf{c}$, $\mathbf{d}$ posterior limb of internal capsule FA value $=0.37$ and centum semiovale FA value $=0.20$. Follow-up examination: e axial T2 showed thinning of the peri-trigonal white matter with approximation of the corticoventricular distance (PVL picture). f Tractography showed mild thinning of the corticospinal tracts in CP infant

measurement of FA values within the posterior limbs of the internal capsules is likely to reduce the possibility of missing these cases and increase the accuracy of MRI in early prediction of pathological outcome namely cerebral palsy.

The main limitation of the current study is represented by the relatively limited number of HIE newborns recruited. The present results should be confirmed in a larger population. For ethical reasons, there was no control group of normal neonates for comparison, as no informed consent could be obtained from parents of normal appearing neonates. Another limitation of this study is noted in most studies of neonatal encephalopathy that exact time of injury was not known in most of the patients. The extent and pattern of injury differ temporally. Particularly in the case of DTI, some regions will 


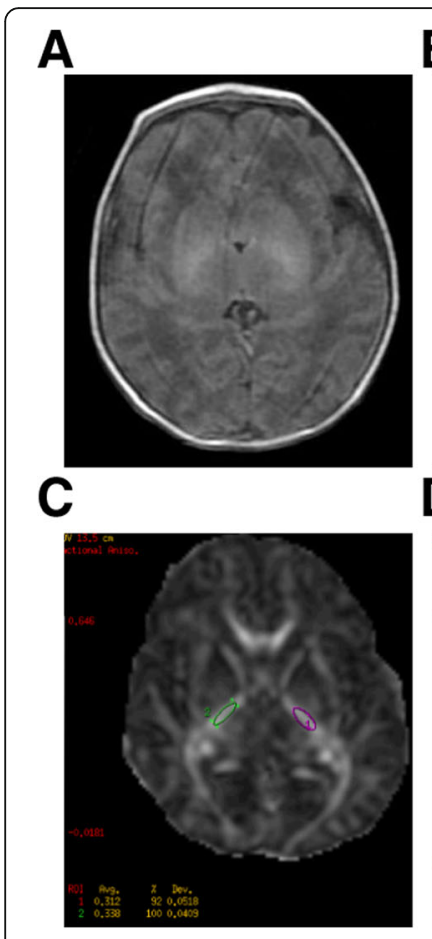

B
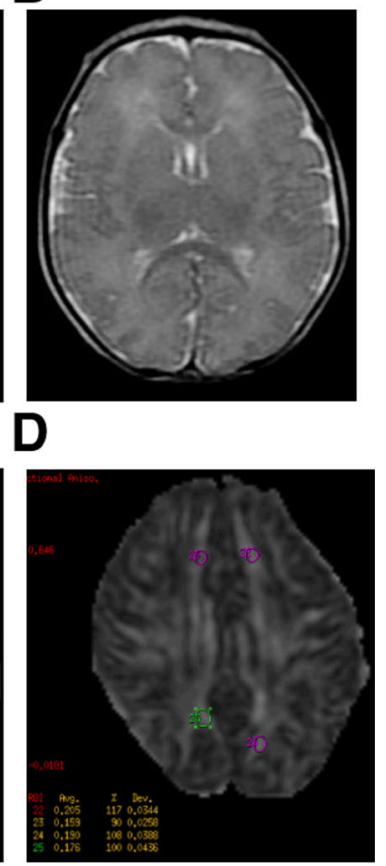

$\mathbf{F}$

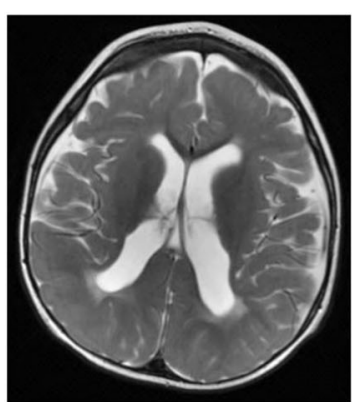

Fig. 4 Eleven days neonate presenting with severe HIE picture, Sarnat III with CP outcome. Conventional MRI: $\mathbf{a}, \mathbf{b}$ axial T1 and T2 showed preserved PLIC signal. DTI: $\mathbf{c}$, d posterior limb of internal capsule FA value $=0.31$ and centum semiovale FA value $=0.20$. Follow-up examination: e axial T2 showed thinning of the peritrigonal white matter with undulated borders of the lateral ventricles (PVL picture). $\mathbf{f}$ Tractography showed mild thinning of the corticospinal tracts in CP infant

appear more severely damaged and some less damaged, depending on the timing of the examination with respect to the injury. Lastly, and to illuminate the full quantitative assessment power of DTI versus the qualitative power of conventional MRI and DWI, extended followup at the age of 2 years (at which complete myelination is acquired) should have been done.

In summary, among imaging-based parameters, DTI values closely correlated with the Bayley scores. Such technique can be carried out in a single individual, which has important clinical significance, as it can accurately and objectively assess the prognosis of full-term neonates with HIE which can guide the treating clinicians for early intervention.

\section{Conclusion}

Application of advanced MRI techniques including DTI in assessment of full-term newborns with perinatal HIE is a more accurate quantitative MR biomarker for prediction of neurodevelopmental outcome mainly cerebral palsy.

\section{Abbreviations}

CP: Cerebral palsy; CSO: Centrum semiovale; CST: Corticospinal tract: DTI: Diffusion tensor imaging; FA: Fractional anisotropy; HIE: Hypoxic ischemic encephalopathy; PLIC: Posterior limb of the internal capsule

\section{Acknowledgements}

Not applicable

\section{Authors' contributions}

$A B$ drafted the manuscript. $A B$ and MA edited the images. TO and $A O$ reviewed the clinical part of the work. AN worked on the conception and oversaw the entire project. All authors read and approved the final manuscript.

\section{Funding}

Not applicable

\section{Availability of data and materials}

The datasets used and/or analyzed during the current study are available from the corresponding author on reasonable request.

\section{Ethics approval and consent to participate}

Approval for this cross-sectional prospective study was obtained from the Research Ethics Committee of Alexandria Faculty of Medicine (Ethics committee's reference number: 020814, IRB no: 00012098, FWA no: 00018699). All study procedures were carried out in accordance with the Declaration of Helsinki regarding research involving human subjects. Written informed consent was obtained from the parents of each child.

\section{Consent for publication}

Written informed consent for publication of this data was given by the parents or legal guardians of the included children.

\section{Competing interests}

The authors declare that they have no competing interests.

\section{Author details}

${ }^{1}$ Department of Radiodiagnosis, Faculty of Medicine, University of Alexandria, Alexandria, Egypt. ${ }^{2}$ Department of Pediatrics, Faculty of Medicine, University of Alexandria, Alexandria, Egypt.

Received: 6 June 2019 Accepted: 5 November 2019

Published online: 02 December 2019

\section{References}

1. Douglas-Escobar M, Weiss MD (2015) Hypoxic-ischemic encephalopathy: a review for the clinician. JAMA Pediatr 169(4):397-403

2. Hagberg $H$, Edwards A, Groenendaal F (2016) Perinatal brain damage: the term infant. Neurobiol Dis 92:102-112

3. Ma H, Sinha B, Pandya RS, Lin N, Popp AJ, Li J et al (2012) Therapeutic hypothermia as a neuroprotective strategy in neonatal hypoxic-ischemic brain injury and traumatic brain injury. Curr Mol Med 12(10):1282-1296

4. Brandon J, Cesar C, Ho WM, Jiping T, John H, Zhang JH (2015) Neuroprotective strategies after neonatal hypoxic ischemic encephalopathy. Int J Mol Sci 16(9):22368-22401

5. Jose A, Matthai J, Paul S (2013) Correlation of EEG, CT, and MRI brain with neurological outcome at 12 months in term newborns with hypoxic ischemic encephalopathy. J Clin Neonatol 2:125-130 
6. Duong $T Q$, Watts $L T$ (2016) A brief report on MRI investigation of experimental traumatic brain injury. Neural Regen Res 11:15-17

7. Goergen SK, Ang H, Wongc F, Carse EA, Charlton M, Evans R et al (2014) Early MRI in term infants with perinatal hypoxic ischemic brain injury: interobserver agreement and MRI predictors of outcome at 2 years. Clinical Radiology 69:72-81

8. Rutherford M, Malamateniou C, McGuinness A, Allsop J, Biarge MM, Counsell S (2010) Magnetic resonance imaging in hypoxic-ischaemic encephalopathy. Early Hum Dev 86(6):351-360

9. Ancora G, Testa C, Grandi S, Tonon C, Sbravati F, Savini S et al (2013) Prognostic value of brain proton MR spectroscopy and diffusion tensor imaging in newbornswith hypoxic ischemic encephalopathy treated by brain cooling. Neuroradiology 55(8):1017-1025

10. Feldman HM, Yeatman JD, Lee ES, Barde LHF, Gaman-Bean S (2010) Diffusion tensor imaging: a review for pediatric researchers and clinicians. J Dev Behav Pediatr 31(4):346-356

11. Lee AY, Shin DG, Park JS, Hong GR, Chang PH, Seo JP et al (2012) Neural tracts injuries in patients with hypoxic ischemic brain injury: diffusion tensor imaging study. Neurosci Lett 528:16-21

12. World Medical Association (2013) World Medical Association Declaration of Helsinki: ethical principles for medical research involving human subjects. JAMA 310(20):2191-2194

13. Sarnat HB, Sarnat MS (1976) Neonatal encephalopathy following fetal distress: a clinical and electroencephalographic study. Arch Neurol 33(10): 696-705

14. Ghei SK, Zan E, Nathan JE, Choudhri A, Tekes A, Huisman TA et al (2014) MR imaging of hypoxic-ischemic injury in term neonates: pearls and pitfalls. Radiographics 34(4):1047-1061

15. Chalak LF, DuPont TL, Sánchez PJ, Lucke A, Heyne RJ, Morriss MC et al (2014) Neurodevelopmental outcomes after hypothermia therapy in the era of Bayley-III. J Perinatol 34(8):629-633

16. Dopwell F, Maypole J, Sinha B, Currier H, DeBassio W, Augustyn M (2017) "More than Meets the Eye": when the neonatal course may impact several years out. J Dev Behav Pediatr 38:44-46

17. Agut T, León M, Rebollo M, Muchart J, Arca G, Garcia-Alix A (2014) Early identification of brain injury in infants with hypoxic ischemic encephalopathy at high risk for severe impairments: accuracy of MB performed in the first days of life. BMC Pediatr 14:177-184

18. Hayakawa K, Koshino S, Tanda K, Nishimura A, Sato O, Morishita H et al (2018) Diffusion pseudonormalization and clinical outcome in term neonates with hypoxic-ischemic encephalopathy. Pediatr Radiol 48(6):865-874

19. Rutherford MA, Pennock JM, Counsell SJ, Mercuri E, Cowan FM, Dubowitz $L M$ et al (1998) Abnormal magnetic resonance signal in the internal capsule predicts poor neurodevelopmental outcome in infants with hypoxicischemic encephalopathy. Pediatrics 102(2 Pt 1):323-328

20. Barkovich AJ, Miller SP, Bartha A, Newton N, Hamrick SE, Mukherjee P et al (2006) MR imaging, MR spectroscopy, and diffusion tensor imaging of sequential studies in neonates with encephalopathy. AJNR Am J Neuroradiol 27(3):533-547

21. Ward P, Counsell S, Allsop J, Cowan F, Shen Y, Edwards D et al (2006) Reduced fractional anisotropy on diffusion tensor magnetic resonance imaging after hypoxic-ischemic encephalopathy. Pediatrics 117(4):619-630

22. Brissaud O, Amirault M, Villega F, Periot O, Chateil JF, Allard M (2010) Efficiency of fractional anisotropy and apparent diffusion coefficient on diffusion tensor imaging in prognosis of neonates with hypoxic-ischemic encephalopathy: a methodologic prospective pilot study. AJNR Am J Neuroradiol 31(2):282-287

23. Lemyre B, Chau V (2018) Hypothermia for newborns with hypoxic-ischemic encephalopathy. Paediatr Child Health. 23(4):285-291

24. Chan KW, Chow AM, Chan KC, Yang J, Wu EX (2010) Magnetic resonance spectroscopy of the brain under mild hypothermia indicates changes in neuroprotection-related metabolites. Neurosci Lett 475(3):150-155

\section{Publisher's Note}

Springer Nature remains neutral with regard to jurisdictional claims in published maps and institutional affiliations.

\section{Submit your manuscript to a SpringerOpen ${ }^{\circ}$ journal and benefit from:}

- Convenient online submission

- Rigorous peer review

- Open access: articles freely available online

- High visibility within the field

- Retaining the copyright to your article

Submit your next manuscript at $\boldsymbol{\nabla}$ springeropen.com 